\title{
A NSGA-II application with different gene expression technologies integration
}

\author{
Daniel Castillo ${ }^{1}$, Juan Manuel Galvez ${ }^{1}$, Olga Valenzuela ${ }^{1}$ and Ignacio Rojas ${ }^{1}$
}

\begin{abstract}
Thanks to genetic expression, biomarkers related to much kind of diseases could be obtained. However, depending on the previous experimental constraints, these biomarkers can become too many, especially if they are intended to be used in a classification process. Multi-Objective Genetic Algorithm (MOGA) has been thought of as a suitable solution to get a tradeoff among candidate biomarkers and classification accuracy. In this sense, genetic expression and Non Sorting Genetic Algorithm (NSGA) are combined in order to obtain the minimum number of possible genes that achieve the maximum classification accuracy. Only with a few genes, the classifier is expected to be more computationally efficient and faster than with all the genes, besides not endangering the final accuracy.
\end{abstract}

Keywords - breast cancer, genetic algorithm, NSGA-II, classification, genes expression, microarray, RNA-Seq, SVM, genes selection

\section{Introduction}

The human genome discovered has exponentially augmented the possibilities of analysis on human genetics through the bioinformatics support. This is so that the biologic knowledge about the human being is increasing due largely to advances in sequencing technologies as microarray or RNAseq.

The analysis of gene expression profiles from these technologies have become fundamental because they allow to identify key genes that are though as potential biomarkers of the analysed disease.

Although sequencing technologies offer the opportunity to see the whole biological system through the quantification of entire human genome, it is intrinsically an intractable problem due to the high dimensionality of the data.

The gene expression quantification in both microarrays and RNA-seq is arranged as massive parallel information, where the set of features (genes) is usually much larger than the number of samples available.

This very high difference among genes and samples is named as the curse of dimensionality [2,9] or more widely well-known in the literature as (NP)-hard problem. The 'large$\mathrm{p}$ small-n' paradigm has been addressed from different approaches in order to reduce its manifestation: on the one hand, through the integration of samples from multiple platforms and technologies; on the other hand, through the selection of relevant genes depending on the pursued objective.

1: Information and Communications Technology Centre (CITIC-UGR). Univeristy of Granada, Spain.
The first option is being developed extensively in the field of bioinformatics, which seeks to expand the total repertoire of samples and achieve a greater statistical significance of the available population sample. Integration can be done at different levels: platforms (Affymetrix, Illumina, etc.), technologies (microarrays, RNA-seq, etc.), omics (genomic, transcriptomic, methylation, etc.).

The second option implies the considerable reduction of the repertoire of genes (usually in the order of thousands) from a selection carried out using machine learning techniques. This selection not only allows to reduce the complexity of the problem, but also maintains the recognition efficiency in addition to eliminating irrelevant or noisy genes. Therefore, genes selection seems to be very valid to reduce the dimensionality, thereby discarding the ambiguous genes for achieving a high classification accuracy [6].

Genetic algorithms (GAs) [12,13] evaluate and evolve the population using machine learning techniques. Thanks to this, the algorithm can produce robust solutions that are important in fields like bioinformatics because real disease and people are involved.

Data reductions techniques are very important when there are gene expression data due to the high dimensionality of the features comparing to the samples available. Therefore, machine learning, GA, feature selection techniques have been very used in the last years to reduce the dimension of feature genes and to avoid the curse of dimension.

Most real-world optimization problem are affected by more than one conflicting objectives. For this cases, there are a kind of GA called Multiple Objectives Genetic Algorithm (MOGA) [11] that use different objectives for search the optimal or sets of optimal solutions (Pareto front). In this sense, the Non-dominated Sorting Genetic Algorithm II (NSGA-II) [4] will be used in this study. This algorithm allows to minimize the number of genes but maximizing the accuracy achieved by this genes in classification.

Before the apply the GA is necessary to extract the data from their respective technologies. Two sequencing technologies has been used to compute the genes expression, which are explained below.

\section{A. Microarray technology}

Microarray is a method that allows the measurement of the value expressions of a large number of genes simultaneously from a collection of microscopic DNA spots attached to a solid surface. This technology is based on the DNA hybridization process, so that DNA is hybridized for each of the spots that represent one gene value expression. Once the 
Proc. of the Sixth Intl. Conf. Advances in Bio-Informatics, Bio-Technology and Environmental Engineering- ABBE 2018 Copyright ( $)$ Institute of Research Engineers and Doctors, USA. All rights reserved.

ISBN: 978-1-63248-148-1 doi: 10.15224/978-1-63248-148-1-05

step is finished with a laser, the expression values are read and written in a file with the extension .CEL.

Once microarray data are available, all of them are processed and filtered from a quality analysis to be later normalized. Once this was done, the last step is the integration of all microarrays. VirtualArray tool [7] has been used for the integration process.

\section{B. RNA-seq technology}

This technology appeared as a revolutionary tool for transcriptome and as a natural evolutionary step in the study of the genome after the massive use of microarray technology. In this sense, one of the most advantageous aspects is that although RNA-seq can be used only for transcriptome profiling, it also can be combined with other functional genomics methods to enhance the analysis of gene expression. Expression is quantified by counting the number of reads mapped to each locus in the transcriptome assembly step. This expression level can be calculated for exons or genes using contigs or reference transcript annotations. These observed RNA-seq read counts have been robustly validated against previous technologies such as microarrays or quantitative polymerase chain reaction (qPCR) [10].

\section{Material and Methods}

The (NP)-hard problem was minimized and approached from a combination of both options: sample integration and gene selection.

All analyzed RNA samples were obtained from NCBI GEO web platform [1]. 108 samples from microarray series and 6 samples from RNA-seq samples were finally integrated.

Table 1 shows a summary about the series used and their origin. As it can be seen, there are series from different countries, and thus there are samples from different ethnic groups. Furthermore, there are different sequencing technologies in the experiment including samples from Affymetrix [5] and Illumina [8]. Moreover, there are data from different generation sequencing. In summary, samples have been integrated from different generation sequencing, technologies, platforms and countries, bringing all of them heterogeneity to the study.

Both microarray and RNA-seq data have passed a strict pipeline. Microarray samples require restrictive quality analysis to discard non-representative samples which took place due to incorrect acquisition, as well as normalization during pre-processing in order to adapt the range of quantification variability of the samples considered.

In our experiment, 98 genes comply the statistical restrictions of logarithmic fold change $(|\log \mathrm{FC} \geq 2|)$ and $\mathrm{p}$ value $\leq 0.001$ to form the final ranking of relevant genes considered as potential biomarkers of the disease. $\log \mathrm{FC}$ represents the difference between breast cancer and control expressed values, whilst p-value represents the probability of obtaining a result equal or higher than what it was observed when the null hypothesis is true.

\begin{tabular}{|c|c|c|c|c|}
\hline Series & Technology & $\begin{array}{c}\text { Quality } \\
\text { Samples }\end{array}$ & $\begin{array}{c}\text { Excl. } \\
\text { Outliers }\end{array}$ & $\begin{array}{c}\text { Samples } \\
\text { Origin }\end{array}$ \\
\hline GSE52712 & Microarray & 19 & 1 & $\begin{array}{c}\text { Manchester } \\
\text { UK }\end{array}$ \\
\hline GSE40987 & Microarray & 10 & 0 & $\begin{array}{c}\text { Boston } \\
\text { USA }\end{array}$ \\
\hline GSE52262 & Microarray & 16 & 0 & $\begin{array}{c}\text { Houston } \\
\text { USA }\end{array}$ \\
\hline GSE12790 & Microarray & 20 & 1 & $\begin{array}{c}\text { San } \\
\text { Francisco } \\
\text { USA }\end{array}$ \\
\hline GSE46834 & Microarray & 8 & 0 & $\begin{array}{c}\text { New York } \\
\text { USA }\end{array}$ \\
\hline GSE68651 & Microarray & 35 & 1 & $\begin{array}{c}\text { Southampton } \\
\text { UK }\end{array}$ \\
\hline GSE78011 & RNA-seq & 3 & 0 & $\begin{array}{c}\text { Louisville } \\
\text { USA }\end{array}$ \\
\hline GSE81593 & RNA-seq & 3 & 0 & $\begin{array}{c}\text { New York } \\
\text { USA }\end{array}$ \\
\hline TOTAL & & 114 & 3 & \\
\hline
\end{tabular}

TABLE I. INPUT SERIES, TECHNOLOGY, NUMBER OF SAMPLES/OUTLIERS AND SAMPLES ORIGIN

Once the 98 expressed genes have been calculated, a GA was used. All the process followed for achieved this is explained in the subsections below.

\section{A. Non-dominated Sorting Genetic Algorithm II (NSGA-II)}

GAs are algorithms for optimization inspired by the biological mechanisms of reproduction and evolution. Normally, GAs try to maximize or minimize an objective using a function, but this is only when the problem have one objective.

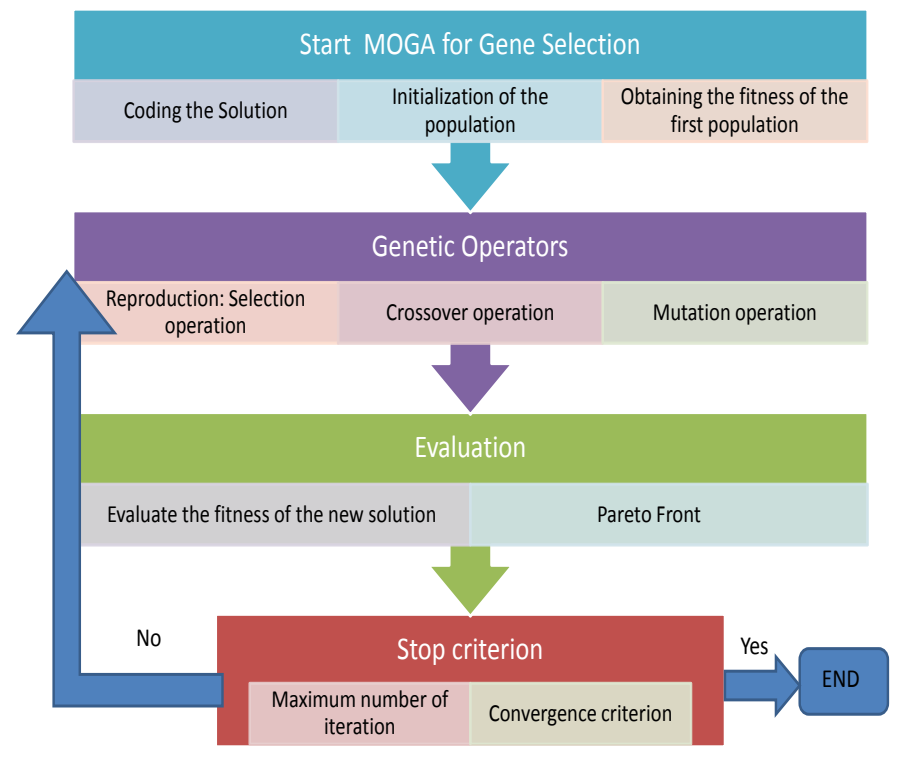

Figure 1. Pipeline followed for feature selection using NSGA-II 
Proc. of the Sixth Intl. Conf. Advances in Bio-Informatics, Bio-Technology and Environmental Engineering- ABBE 2018 Copyright ( $)$ Institute of Research Engineers and Doctors, USA. All rights reserved.

ISBN: 978-1-63248-148-1 doi: 10.15224/978-1-63248-148-1-05

In the case of this study talks about a multi-objective problem due to is necessary minimize the number of genes but maximizing the final accuracy achieved when a classifier is used with these genes.

Hence, NSGA-II have been used. This GA allows reach the optimal solutions of a optimization situation when a multiobjective problem appears and calculated a Pareto front or optimal non-dominated solutions front. So, if a point is better than other points in all of objectives, this is non-dominated by these worst points. The non-dominated points of the last generations make the optimal Pareto front. All the intermediate process is explained then (the block diagram is presented in Fig.1).

First, the initial population is generate with the fixedlength binary strings for $\mathrm{N}$ individuals (the binary codification is presented in Fig.2) .

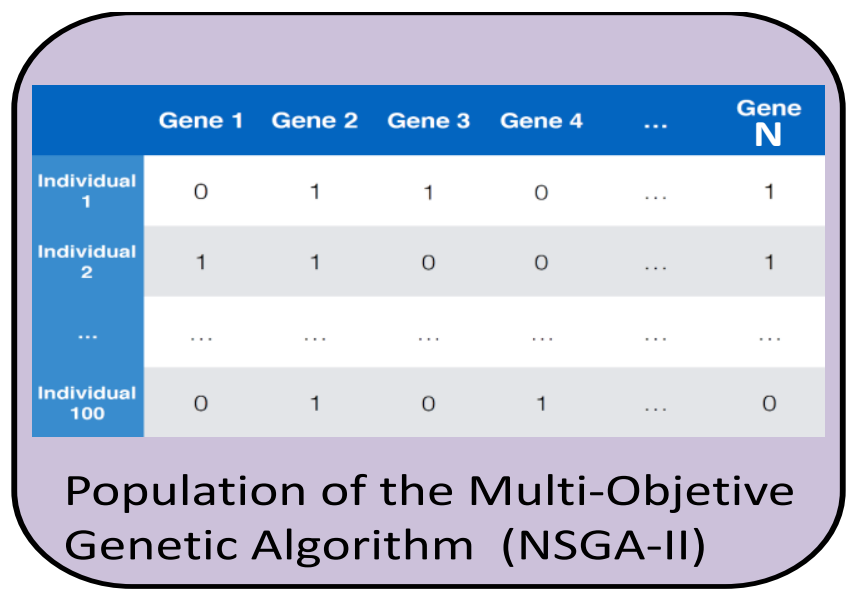

Figure 2. Binary codification of the NSGA-II for selecting the most relevant genes

Each string shows a feature subset and each position in the string are coded as one in this case if the gene have been selected or zero if not. The next step is calculate the fitness for the survival of each feature subset. Such as it said before, two objective will be used so there are two fitness functions, one for minimize the genes and other for maximize the accuracy. The best subsets will be selected for the crossover or mutation for the next generation. The mutation changes some of the values in a subset randomly. In the other hand, crossover join different features from a two subsets (parents) into a new subset (child). This process will be repeat in an iterative process until the maximum number of generation will be reached or until a stop criterion will be achieved.

\section{B. Support Vector Machine (SVM)}

For calculated the second objective function, a SVM has been used in order to obtain the accuracy with the selected genes of the first objective functions. This algorithm is based on the idea of separating the different categories in a problem through a hyperplane. The algorithm calculates the maximum- margin hyperplane that maximizes the distance between different classes. In one dimension, this hyperplane would be a single point. With two dimensions, a line, and with three dimensions a plane would be needed in order to separate the classes. This model could be extrapolated mathematically to higher dimensions [14].

The Fig. 3 shows the pipeline followed for this study.

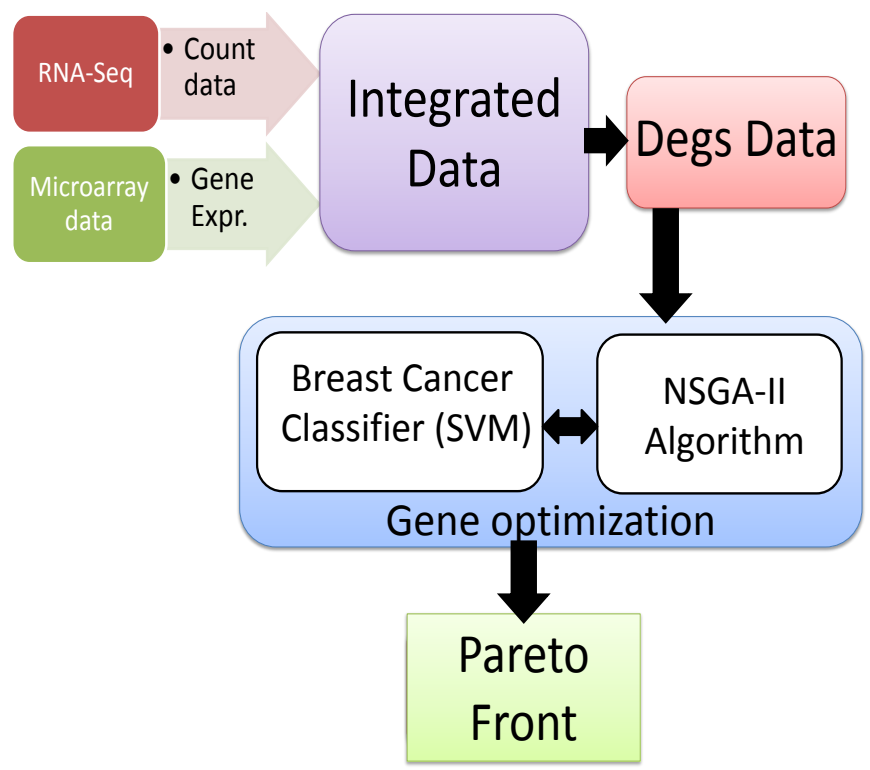

Figure 3. Pipeline followed for simultaneously using microarray data and RNA-Seq data, in conjuntion with NSGA-II, for selecting the most representative genes in breast cancer

\section{Results and Discussion}

The final set of 98 expressed genes have been analysed. An exhaustive list is shown in Table 2. At this point, it should be remembered that the 98 genes shown in this table correspond to those genes that appeared as differentially expressed from the analysis of the complete dataset.

This table is formed by five statistics values computed by the limma package from Bioconductor [3]. The log-fold change $(\log \mathrm{FC})$ represents the difference between breast cancer and control expressed values. If $|\log \mathrm{FC} \geq 2|$ it means that there exists significance differences between cancer and control values. The second value in Table 2 is the the moderated t-statistic, which has the same interpretation as the normal t-statistic but the standard errors have been reduced between the genes, effectively obtaining information from the set of genes to help with inference about each individual gene. The next value is the P-Value (P.Val) which represents the probability of obtaining a result equal or higher than what it was observed when the null hypothesis is true. The B-statistic (B) is the log-odds that a given gene is deferentially expressed.

\begin{tabular}{|c|c|c|c|c|}
\hline $\begin{array}{c}\text { Genes } \\
\text { names }\end{array}$ & $\mid \boldsymbol{l o} \boldsymbol{g} \boldsymbol{F} \boldsymbol{C |} \geq \mathbf{2}$ & $\mathbf{t}$ & p-val & B \\
\hline KRT19 & 7.993 & 11.072 & $8.124 \mathrm{E}-21$ & 36.607 \\
\hline KRT6A & -7.800 & -13.558 & $3.347 \mathrm{E}-27$ & 51.214 \\
\hline NNMT & -7.584 & -11.544 & $4.951 \mathrm{E}-22$ & 39.384 \\
\hline
\end{tabular}


Proc. of the Sixth Intl. Conf. Advances in Bio-Informatics, Bio-Technology and Environmental Engineering- ABBE 2018 Copyright (C) Institute of Research Engineers and Doctors, USA. All rights reserved.

ISBN: 978-1-63248-148-1 doi: 10.15224/978-1-63248-148-1-05

\begin{tabular}{|c|c|c|c|c|}
\hline VIM & -7.261 & -15.117 & $3.917 \mathrm{E}-31$ & 60.213 \\
\hline AKR1B1 & -6.943 & -11.437 & $9.357 \mathrm{E}-22$ & 38.753 \\
\hline SFRP1 & -6.866 & -18.820 & $4.925 \mathrm{E}-40$ & 80.570 \\
\hline TGFBI & -6.701 & -14.299 & $4.424 \mathrm{E}-29$ & 55.515 \\
\hline MT1E & -6.650 & -15.281 & $1.537 \mathrm{E}-31$ & 61.142 \\
\hline C3 & -6.569 & -15.928 & $3.857 \mathrm{E}-33$ & 64.805 \\
\hline BMP7 & 6.406 & 13.058 & $6.330 \mathrm{E}-26$ & 48.292 \\
\hline KRT5 & -6.229 & -9.125 & $7.460 \mathrm{E}-16$ & 25.273 \\
\hline CXCL1 & -6.145 & -13.526 & $4.030 \mathrm{E}-27$ & 51.030 \\
\hline S100A2 & -6.016 & -9.582 & $5.249 \mathrm{E}-17$ & 27.902 \\
\hline KRT7 & -5.991 & -11.975 & $3.850 \mathrm{E}-23$ & 41.922 \\
\hline TNS4 & -5.866 & -25.125 & $1.651 \mathrm{E}-53$ & 111.284 \\
\hline EEF1A2 & 5.764 & 8.956 & $1.979 \mathrm{E}-15$ & 24.307 \\
\hline CLMP & -5.631 & -11.238 & $3.037 \mathrm{E}-21$ & 37.583 \\
\hline IFI16 & -5.543 & -9.230 & $4.073 \mathrm{E}-16$ & 25.872 \\
\hline LAMC2 & -5.426 & -12.346 & $4.247 \mathrm{E}-24$ & 44.112 \\
\hline IGFBP4 & 5.412 & 13.779 & $9.173 \mathrm{E}-28$ & 52.501 \\
\hline FAM83A & -5.328 & -14.042 & $1.974 \mathrm{E}-28$ & 54.028 \\
\hline SYTL2 & 5.283 & 11.883 & $6.617 \mathrm{E}-23$ & 41.384 \\
\hline SNAI2 & -5.169 & -9.731 & $2.204 \mathrm{E}-17$ & 28.762 \\
\hline DNER & -5.152 & -11.859 & $7.620 \mathrm{E}-23$ & 41.244 \\
\hline PRKCDBP & -5.105 & -10.241 & $1.105 \mathrm{E}-18$ & 31.730 \\
\hline ALOX15B & -5.088 & -16.524 & $1.353 \mathrm{E}-34$ & 68.133 \\
\hline IGFBP5 & 5.085 & 8.165 & $1.755 \mathrm{E}-13$ & 19.871 \\
\hline $\mathrm{BNC1}$ & -5.072 & -16.335 & $3.889 \mathrm{E}-34$ & 67.085 \\
\hline GFRA1 & 5.021 & 6.872 & $1.958 \mathrm{E}-10$ & 12.955 \\
\hline DSC3 & -4.999 & -17.145 & $4.296 \mathrm{E}-36$ & 71.561 \\
\hline PTGES & -4.990 & -17.489 & $6.479 \mathrm{E}-37$ & 73.440 \\
\hline TFF1 & 4.925 & 4.857 & $3.168 \mathrm{E}-06$ & 3.497 \\
\hline RAB25 & 4.864 & 8.521 & $2.368 \mathrm{E}-14$ & 21.851 \\
\hline KRT14 & -4.863 & -6.445 & $1.768 \mathrm{E}-09$ & 10.794 \\
\hline EFEMP1 & -4.855 & -10.020 & $4.059 \mathrm{E}-18$ & 30.440 \\
\hline SLPI & -4.793 & -10.194 & $1.455 \mathrm{E}-18$ & 31.457 \\
\hline SDPR & -4.728 & -12.002 & $3.264 \mathrm{E}-23$ & 42.086 \\
\hline FBP1 & 4.707 & 6.789 & $3.017 \mathrm{E}-10$ & 12.530 \\
\hline EPCAM & 4.662 & 8.150 & $1.906 \mathrm{E}-13$ & 19.790 \\
\hline GNA15 & -4.570 & -15.676 & $1.614 \mathrm{E}-32$ & 63.382 \\
\hline HTRA1 & -4.527 & -10.906 & $2.178 \mathrm{E}-20$ & 35.627 \\
\hline RAC2 & -4.524 & -11.727 & $1.669 \mathrm{E}-22$ & 40.465 \\
\hline CLCA2 & -4.411 & -9.272 & $3.189 \mathrm{E}-16$ & 26.115 \\
\hline GPX1 & -4.384 & -6.773 & $3.281 \mathrm{E}-10$ & 12.448 \\
\hline EMP3 & -4.383 & -9.299 & $2.728 \mathrm{E}-16$ & 26.269 \\
\hline SERPINB5 & -4.371 & -8.314 & $7.600 \mathrm{E}-14$ & 20.698 \\
\hline TSPYL5 & 4.317 & 6.297 & $3.735 \mathrm{E}-09$ & 10.062 \\
\hline GSTP1 & -4.242 & -5.846 & $3.433 \mathrm{E}-08$ & 7.892 \\
\hline SLC2A10 & 4.216 & 11.411 & $1.088 \mathrm{E}-21$ & 38.602 \\
\hline LDHB & -4.182 & -5.892 & $2.745 \mathrm{E}-08$ & 8.111 \\
\hline VSTM2L & -4.146 & -11.277 & $2.409 \mathrm{E}-21$ & 37.813 \\
\hline BIRC3 & -4.079 & -13.064 & $6.110 \mathrm{E}-26$ & 48.327 \\
\hline ABLIM3 & -4.000 & -12.337 & $4.481 \mathrm{E}-24$ & 44.059 \\
\hline TFCP2L1 & -3.874 & -11.847 & $8.202 \mathrm{E}-23$ & 41.171 \\
\hline DSG3 & -3.820 & -8.387 & $5.035 \mathrm{E}-14$ & 21.105 \\
\hline SLC26A2 & -3.798 & -13.491 & $4.947 \mathrm{E}-27$ & 50.826 \\
\hline C3orf14 & 3.763 & 7.772 & $1.558 \mathrm{E}-12$ & 17.715 \\
\hline IL20RB & -3.667 & -8.868 & $3.262 \mathrm{E}-15$ & 23.812 \\
\hline FXYD5 & -3.623 & -5.585 & $1.191 \mathrm{E}-07$ & 6.679 \\
\hline GSTM3 & 3.590 & 9.622 & $4.161 \mathrm{E}-17$ & 28.133 \\
\hline ADRB2 & -3.572 & -9.968 & $5.512 \mathrm{E}-18$ & 30.136 \\
\hline EMP1 & -3.535 & -7.622 & $3.543 \mathrm{E}-12$ & 16.905 \\
\hline IGFBP7 & -3.530 & -4.676 & $6.866 \mathrm{E}-06$ & 2.751 \\
\hline GJB5 & -3.517 & -12.456 & $2.225 \mathrm{E}-24$ & 44.755 \\
\hline HENMT1 & 3.514 & 7.953 & $5.732 \mathrm{E}-13$ & 18.702 \\
\hline ZBED2 & -3.507 & -6.452 & $1.705 \mathrm{E}-09$ & 10.830 \\
\hline MSLN & -3.504 & -8.558 & $1.917 \mathrm{E}-14$ & 22.061 \\
\hline IL18 & -3.415 & -9.270 & $3.223 \mathrm{E}-16$ & 26.104 \\
\hline TRIM29 & -3.395 & -9.588 & $5.081 \mathrm{E}-17$ & 27.934 \\
\hline OSR2 & 3.346 & 8.380 & $5.238 \mathrm{E}-14$ & 21.066 \\
\hline LAMB1 & -3.346 & -6.972 & $1.162 \mathrm{E}-10$ & 13.468 \\
\hline
\end{tabular}

\begin{tabular}{|c|c|c|c|c|}
\hline UCP2 & 3.332 & 5.788 & $4.539 \mathrm{E}-08$ & 7.620 \\
\hline CPVL & -3.331 & -7.870 & $9.043 \mathrm{E}-13$ & 18.253 \\
\hline KRT81 & -3.320 & -5.133 & $9.424 \mathrm{E}-07$ & 4.670 \\
\hline S100A8 & -3.292 & -5.698 & $6.982 \mathrm{E}-08$ & 7.200 \\
\hline TP5313 & -3.242 & -11.149 & $5.160 \mathrm{E}-21$ & 37.057 \\
\hline FOXA1 & 3.226 & 5.576 & $1.241 \mathrm{E}-07$ & 6.640 \\
\hline SLC24A3 & 3.211 & 6.190 & $6.356 \mathrm{E}-09$ & 9.541 \\
\hline PNLIPRP3 & -3.200 & -7.998 & $4.470 \mathrm{E}-13$ & 18.948 \\
\hline INHBB & 3.180 & 7.756 & $1.698 \mathrm{E}-12$ & 17.630 \\
\hline RAB38 & -3.129 & -9.539 & $6.781 \mathrm{E}-17$ & 27.649 \\
\hline ZBTB16 & -3.112 & -8.869 & $3.251 \mathrm{E}-15$ & 23.816 \\
\hline PLD5 & -3.070 & -11.039 & $9.925 \mathrm{E}-21$ & 36.408 \\
\hline DFNA5 & -3.047 & -7.565 & $4.835 \mathrm{E}-12$ & 16.599 \\
\hline FKBP5 & -2.988 & -10.435 & $3.528 \mathrm{E}-19$ & 32.863 \\
\hline CD109 & -2.986 & -7.196 & $3.541 \mathrm{E}-11$ & 14.637 \\
\hline CASP1 & -2.955 & -6.388 & $2.367 \mathrm{E}-09$ & 10.509 \\
\hline SULT1E1 & -2.903 & -7.749 & $1.763 \mathrm{E}-12$ & 17.594 \\
\hline FAM174B & 2.779 & 5.557 & $1.353 \mathrm{E}-07$ & 6.555 \\
\hline PDZK1IP1 & -2.752 & -7.028 & $8.611 \mathrm{E}-11$ & 13.743 \\
\hline TNNI2 & -2.750 & -7.896 & $7.842 \mathrm{E}-13$ & 18.393 \\
\hline CAV1 & -2.727 & -5.028 & $1.503 \mathrm{E}-06$ & 4.217 \\
\hline IRX4 & -2.714 & -7.628 & $3.433 \mathrm{E}-12$ & 16.936 \\
\hline KRT80 & 2.706 & 5.268 & $5.131 \mathrm{E}-07$ & 5.259 \\
\hline FOXO1 & -2.649 & -8.921 & $2.408 \mathrm{E}-15$ & 24.113 \\
\hline SNCA & -2.635 & -8.533 & $2.211 \mathrm{E}-14$ & 21.919 \\
\hline TBL1X & 2.565 & 9.676 & $3.043 \mathrm{E}-17$ & 28.442
\end{tabular}

TABLE II. List of 98 expressed genes obtained with limma as the intersection of microarray, RNA-Seq and integrated dataset

Such as said before, two objective functions have been used in order to achieve the optimal solution for the problem. The first function is the number of active chromosome genes, in this case the number of genes used for the classification. The second function is the accuracy reached with the number of genes selected by the first function. So, the GA will minimize the number of selected genes but maximizing the accuracy achieved.

In this sense, the chromosome have 98 genes because the study has 98 expressed genes and an initial population of 200 individuals. Furthermore, the GA has been done crossover between the population during 40 generations with a crossover rate equal to 0.7 and a mutation probability of 0.1 .

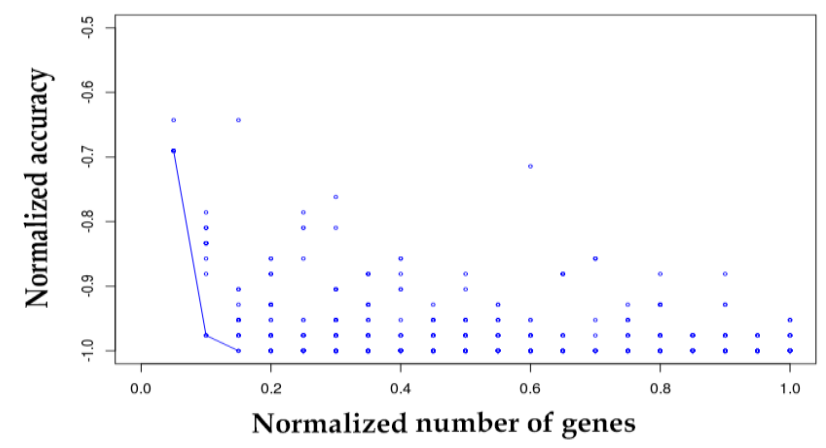

Figure 4. Optimal Pareto front calculated by the Genetic Algorithm NSGA-II

The result can be seen at the Fig. 2. Three non-dominant points appear in the plot, these points show the best solutions for the problem of this study called Pareto front. This 
representation has one point with one gene, one point with two genes and one point with three genes. This last point have two overlapped points because there are two combinations of three genes that achieved the maximum accuracy. These genes and their results are shown by the Table 3. Also, the expression levels of these genes for cancer and control samples have been represented at the Fig. 4. The values show that all genes except one of them (IFI16) have the expression levels with many differentiation between cancer and control samples. This means that they are good biomarkers for breast cancer classification.

\begin{tabular}{|c|c|}
\hline Gene Names & Accuracy \\
\hline PTGES & $69.047 \%$ \\
\hline DSC3, SLC26A2 & $97.619 \%$ \\
\hline FKBP5, GSTM3, IFI16 & $100 \%$ \\
\hline INHBB, PLD5, PTGES & $100 \%$ \\
\hline $\begin{array}{c}\text { TABLE III. Optimum genes calculated by the GA for achieving the } \\
\text { maximum accuracy }\end{array}$
\end{tabular}
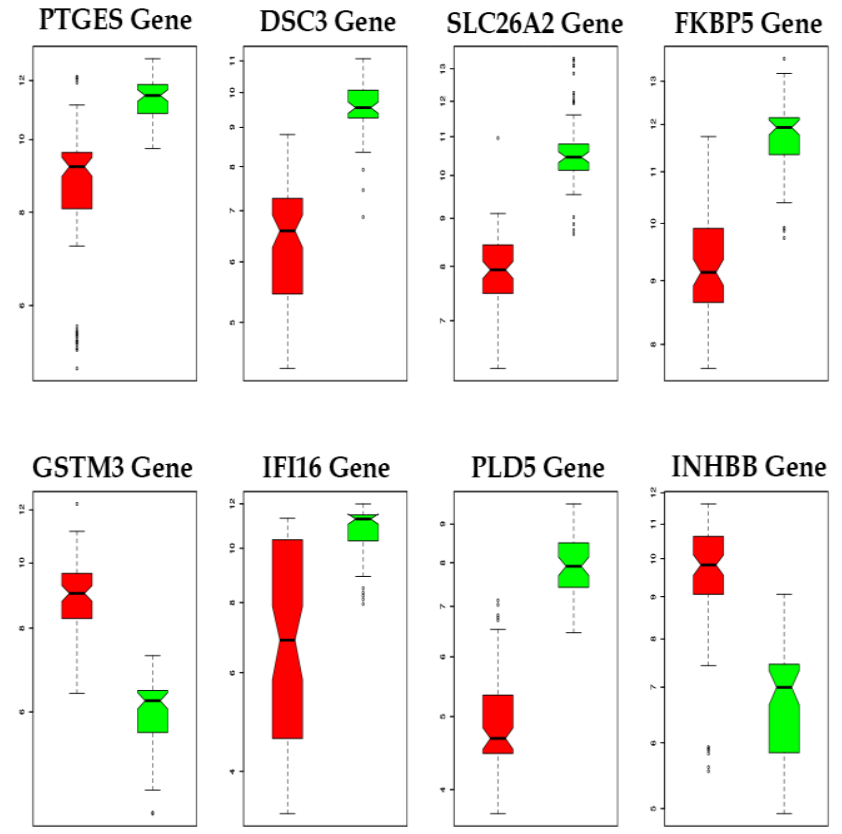

Figure 5.

Expression levels of the genes that appear in Pareto front

\section{Conclusions}

An heterogeneous data integration from different technologies (microarray and RNA-seq) that quantify the quantity of RNA in human biological samples is carried out in this work.

Once this integration was done 98 expressed genes that allow difference between breast cancer and control samples were achieved.

A multi-objective genetic algorithm (NSGA-II) has been used in order to minimize the number of genes needed in the classification process but without endanger the accuracy.
Finally, a Pareto front with 4 non dominated point has been calculated. This points ensures the maximum accuracy but minimizing the number of genes. Only with one genes the accuracy is $70 \%$, but the most important question is that only using two or three genes the final accuracy is between 971\% and $100 \%$, so the main purpose of this study have been achieved and the fact of use a GA is a great way of reduce the dimensionality of the feature in biological data like genes. The most important novelty is apply the GA to an integrated dataset from different technologies like microarray and RNAseq.

\section{Acknowledgment}

This work has been partially supported by the project from the Ministry of Spain with the reference TIN2015-71873 and from J. Andalucia with reference P12-TIC-2082.

\section{References}

[1] Tanya Barre., Dennis B Troup, Stephen E Wilhite, Pierre Ledoux, Dmitry Rudnev, Carlos Evangelista, Irene F Kim, Alexandra Soboleva, Maxim Tomashevsky, and Ron Edgar. 2007. NCBI GEO: mining tens of millions of expression profiles database and tools update. Nucleic acids research 35, suppl 1 (2007), D760-D765.

[2] JM Bernardo, MJ Bayarri, JO Berger, AP Dawid, D Heckerman, AFM Smith, and M West. 2003. Bayesian factor regression models in the €large p, small nfi paradigm. Bayesian statistics 7 (2003), 733-742.

[3] BioConductor. 2004. Limma moderated t-statistics and B-statistics. (2004). h.ps://stat.ethz.ch/pipermail/bioconductor/2004September/006132.html

[4] Kalyanmoy Deb, Amrit Pratap, Sameer Agarwal, and TAMT Meyarivan. 2002. A fast and elitist multiobjective genetic algorithm: NSGA-II. IEEE transactions on evolutionary computation 6, 2 (2002), 182-197.

[5] Hinrich Gohlmann and Willem Talloen. 2009. Gene expression studies using Affymetrix microarrays. CRC Press.

[6] Isabelle Guyon, Jason Weston, Stephen Barnhill, and Vladimir Vapnik. 2002. Gene selection for cancer classifcation using support vector machines. Machine learning 46, 1 (2002), 389-422.

[7] Andreas Heider and R"udiger Alt. 2013. virtualArray: a R/bioconductor package to merge raw data from di.erent microarray platforms. BMC bioinformatics 14, 1 (2013), 75.

[8] Illumina. 2009. Illumina Genes Expression arrays. (2009). h.p://www.illumina.com/techniques/microarrays/gene-expressionarrays.html

[9] Patrenahalli M. Narendra and Keinosuke Fukunaga. 1977. A branch and bound algorithm for feature subset selection. IEEE Trans. Comput. 26, 9 (1977), 917-922.

[10] Stuart N Peirson and Jason N Butler. 2007. .antitative polymerase chain reaction. Circadian Rhythms: Methods and Protocols (2007), 349-362.

[11] Silvia Poles. 2003. MOGA-II an improved multi-objective genetic algorithm. ESTECO-Technical Report (2003).

[12] Feng Tan, Xuezheng Fu, Yanqing Zhang, and Anu G Bourgeois. 2006. Improving feature subset selection using a genetic algorithm for microarray gene expression data. In Evolutionary Computation, 2006. CEC 2006. IEEE Congress on. IEEE, 2529-2534.

[13] Wang Wei and Liu Hong. 2010. Genetic algorithm and support vector machinebased gene microarray analysis*,.. Journal of Clinical Rehabilitative Tissue Engineering Research 14, 17 (2010), 3099-3103.

[14] Cortes, C., \& Vapnik, V. (1995). Support-vector networks. Machine learning, 20(3), 273-297. 